\title{
EFFECTS OF MICRONUTRIENT APPLICATION ON DIFFERENT ATTRIBUTES OF POTATO IN FLOODPLAIN SOILS OF BANGLADESH
}

\author{
M.M.H. Sarker ${ }^{\text {** A.Z.M Moslehuddin }}{ }^{2}$, M. Jahiruddin ${ }^{2}$ and M.R. Islam ${ }^{2}$ \\ ${ }^{1}$ Department of Soil Science, Sylhet Agricultural University, Sylhet-3100, Bangladesh \\ ${ }^{2}$ Department of Soil Science, Bangladesh Agricultural University \\ Mymensingh-2202, Bangladesh
}

\begin{abstract}
Different crops have variations in their responses to applied micronutrients in soil. A study was conducted on floodplain soil of Bangladesh to explore the response of potato to application of micronutrients in soil. The experimental site was located at farmers' field in Chandina upazila under Cumilla district of Bangladesh covering the soils of Old Meghna Estuarine Floodplain (AEZ 19) during 2011-12. Randomized complete block design with 3 replications of each treatment was used in the experiment, where seven treatments including a control were tested. Additive element trial technique was followed while designing the treatments taking six micronutrients i. e. $\mathrm{Zn}, \mathrm{B}, \mathrm{Cu}, \mathrm{Mn}, \mathrm{Fe}$ and $\mathrm{Mo}$ at the rate of $3,2,2,3,5$ and $1 \mathrm{~kg} \mathrm{ha}^{-1}$, respectively. Macronutrients, such as $\mathrm{N}, \mathrm{P}, \mathrm{K}$ and $\mathrm{S}$ were applied at recommended rates to all plots. The highest tuber yield $\left(28.7 \mathrm{t} \mathrm{ha}^{-1}\right)$ was produced by the combined application of $\mathrm{Zn}$ and B. Only $\mathrm{Zn}$ application was sufficient to obtain the highest content of protein as well as content of almost all the nutrients in potato tuber. Antagonistic relation between $\mathrm{Zn}$ and $\mathrm{P}$ in soil-plant system was recorded in the study. Zinc and boron application influenced different growth and yield parameters of potato while the other four added micronutrients did not have any significant effect but combined application of $\mathrm{Zn}, \mathrm{B}, \mathrm{Cu}, \mathrm{Mn}, \mathrm{Fe}$ and Mo had beneficial role for better plant growth and production. Proper management of zinc and boron fertilizers including optimization of application rates of those nutrients can help to uphold the yield and quality of potato in floodplain soil.
\end{abstract}

Keywords: Micronutrients, potato, floodplain soil, Bangladesh.

\footnotetext{
*Corresponding author email: mosharaf_srdi@yahoo.com, mosharaf_soil@sau.ac.bd
} 
M.M.H. Sarker et al.

\section{INTRODUCTION}

The agro-based economy of Bangladesh has two main challenges which are vast population to feed and small arable land area. To produce more food for the ever increasing population the arable land is being intensively used. Cropping intensity of this country in 1983-84 was $171 \%$ which has become $194 \%$ in $2015-16$ (BBS, 2017). Moreover, cultivation of HYV and hybrid varieties of different crops is deteriorating soil fertility day by day due to exhaustive nature of those varieties. As a consequence new nutrient deficiency in soil is emerging. Chronologically N, P, K, S, Zn and B deficiencies have arisen in this country's soils (Islam, 2008). Occurrence of $\mathrm{Cu}, \mathrm{Mo}$ and $\mathrm{Mn}$ deficiencies in crops are reported sporadically (Bhuiyan et al., 1998 and Khanam et al., 2000). Some reasons of micronutrient deficiency in Bangladesh were highlighted by Jahiruddin and Islam (2014) and those are organic matter depletion, unbalanced use of fertilizers, minimum or no use of manure, high cropping intensity, high $\mathrm{pH}$ (e.g. calcareous soils), nutrient leaching and light textured soils (Jahiruddin and Islam, 2014). Farmers of Bangladesh are not habituated with the use of micronutrient in crop cultivation that challenge balanced fertilization and creates negative impact in crop production (Rijpma and Jahiruddin, 2004).

Micronutrients help increase the efficiency of the use of macronutrients. Again, continual use of micronutrients may lead to an accumulation of toxic levels of those that may threaten crop quality. Hence, judicious application of micronutrients is very much essential; whereas micronutrients have received less attention in different research and extension projects. Different institutions have carried out a number of field trials with micronutrients at different regions of the country. These researches were concentrated mainly on cereal crops, but those were scanty with vegetables. Experiments with micronutrient have been conducted mainly on rice (Jahiruddin et al., 1994), wheat (Hossain, 2005) and maize (Alam et al., 2000), among the cereals. Among the vegetables, potatoes have been an important constituent of food for centuries and are an integral part of the diet, both for rich and poor population. Some field trials on micronutrients in vegetables cultivation have been made (Nasreen et al., 2009).

Impact of a micronutrient deficiency is commonly measured as loss of crop yield; nevertheless quality of harvested products is also important. For the sake of improved human and animal health, micronutrient levels in foods need to be enhanced. Bell and Dell (2008) estimated that more than three billion people in the world are suffering from micronutrient malnutrition. A nutrient balanced diet is the aim of any sustainable food security program. Studies have revealed that micronutrient deficiency led disorders occur in over half of the total human population globally. As per available literature, an adult human body has about 2-3 g of zinc, about $0.1 \%$ of which is replenished daily. The recent studies in molecular physiology strongly suggest that in some cases the iron deficiencies in humans may be associated with zinc deficiency (Upadhayay et al., 2012). The soil resource of Bangladesh mostly comprises with floodplain soils and the Old Meghna Estuarine Floodplain (AEZ 19) 
has coverage of huge agricultural land area. With a view of considering the above points, a study was conducted for evaluating the effect of micronutrient application on different traits of potato in floodplain soil of Bangladesh.

\section{MATERIALS AND METHODS}

\section{Experimental site}

Farmer's field in Gabura village under Chandina upazila of Cumilla district in Bangladesh was the site of the experiment. The soil of the experimental field belonged to the Old Meghna Estuarine Floodplain (AEZ 19) having Chandina soil series under Non-Calcareous Dark Grey Floodplain Soil type. The nutrient status including other parameters in initial soil is shown in Table 1. The popular potato variety 'Diamant' was used in the trial.

Table 1. Initial status of nutrients and other parameters in soil of experimental field

\begin{tabular}{|c|c|}
\hline Characteristics & Status \\
\hline Organic matter $(\%)$ & 1.68 \\
\hline $\mathrm{pH}$ & 6.1 \\
\hline Total N (\%) & 0.10 \\
\hline Available $\mathrm{P}\left(\mathrm{mg} \mathrm{kg}^{-1}\right)$ & 14.8 \\
\hline Exchangeable $\mathrm{K}\left(\mathrm{cmol}_{\mathrm{c}} \mathrm{kg}^{-1}\right)$ & 0.07 \\
\hline Exchangeable $\mathrm{Ca}\left(\mathrm{cmol}_{\mathrm{c}} \mathrm{kg}^{-1}\right)$ & 3.95 \\
\hline Exchangeable $\mathrm{Mg}\left(\mathrm{cmol}_{\mathrm{c}} \mathrm{kg}^{-1}\right)$ & 2.73 \\
\hline Available $\mathrm{S}\left(\mathrm{mg} \mathrm{kg}^{-1}\right)$ & 7.5 \\
\hline Available $\mathrm{Zn}\left(\mathrm{mg} \mathrm{kg}^{-1}\right)$ & 0.79 \\
\hline Available B ( $\left.\mathrm{mg} \mathrm{kg}^{-1}\right)$ & 0.28 \\
\hline Available $\mathrm{Cu}\left(\mathrm{mg} \mathrm{kg}^{-1}\right)$ & 2.28 \\
\hline Available $\mathrm{Fe}\left(\mathrm{mg} \mathrm{kg}^{-1}\right)$ & 257 \\
\hline Available $\mathrm{Mn}\left(\mathrm{mg} \mathrm{kg}^{-1}\right)$ & 8.9 \\
\hline
\end{tabular}

\section{Treatments}

Seven treatment combinations including a control were tested in the experiment. The treatments were $\mathrm{T}_{1}(\mathrm{Control}), \mathrm{T}_{2}(\mathrm{Zn}), \mathrm{T}_{3}(\mathrm{Zn}+\mathrm{B}), \mathrm{T}_{4}(\mathrm{Zn}+\mathrm{B}+\mathrm{Cu}), \mathrm{T}_{5}(\mathrm{Zn}+\mathrm{B}+\mathrm{Cu}+\mathrm{Mn})$, $\mathrm{T}_{6}(\mathrm{Zn}+\mathrm{B}+\mathrm{Cu}+\mathrm{Mn}+\mathrm{Fe})$ and $\mathrm{T}_{7}(\mathrm{Zn}+\mathrm{B}+\mathrm{Cu}+\mathrm{Mn}+\mathrm{Fe}+\mathrm{Mo})$. Micronutrients were 
applied as $\mathrm{ZnSO}_{4} .7 \mathrm{H}_{2} \mathrm{O}, \mathrm{H}_{3} \mathrm{BO}_{3}, \mathrm{CuSO}_{4} .5 \mathrm{H}_{2} \mathrm{O}, \mathrm{MnCl}_{2}, \mathrm{FeSO}_{4} .7 \mathrm{H}_{2} \mathrm{O}$ and $\mathrm{Na}_{2} \mathrm{MoO}_{4}$ at the rates of $3 \mathrm{~kg} \mathrm{Zn}, 2 \mathrm{~kg} \mathrm{~B}, 2 \mathrm{~kg} \mathrm{Cu}, 3 \mathrm{~kg} \mathrm{Mn}, 5 \mathrm{~kg} \mathrm{Fe}$ and $1 \mathrm{~kg} \mathrm{Mo} \mathrm{ha}{ }^{-1}$, respectively. To apply different macronutrients, urea, TSP, MoP and gypsum were used at recommended rates $\left(125,25,100\right.$ and $10 \mathrm{~kg} \mathrm{ha}^{-1}$ for $\mathrm{N}, \mathrm{P}, \mathrm{K}$ and $\mathrm{S}$, respectively) equally for all plots (FRG, 2005).

\section{Experimental design and layout}

The experiment was set up with randomized complete block design having three replications of each treatment. The treatments were randomly distributed to the plots in each block. The individual plots measuring $5 \mathrm{~m} \times 4$ m were surrounded by $40 \mathrm{~cm}$ wide and $10 \mathrm{~cm}$ height earthen bunds. One meter wide and $10 \mathrm{~cm}$ deep irrigation channel was made in-between two blocks. The land was prepared well before planting. The layout of the experiment and randomization was done in accordance with the standard statistical methods.

\section{Planting potato seeds and intercultural operations}

Furrows were made with a plough and sprouted seed tubers were planted in the furrows maintaining a spacing $55 \mathrm{~cm} \times 15 \mathrm{~cm}$. All the tubers in the furrows were covered with soil. The first earthing-up was done after the second dose of urea application at 30 DAP. The $2^{\text {nd }}$ earthing-up was done at 40 DAP. Irrigation was provided once at 35 DAP. To control late blight disease, ridomyl was sprayed at 10day intervals starting from 25 DAP until maturity. To control rodent, phostoxin tablet (fumigant) was inserted into the hole of the rodents and then opening of the hole was blocked with soil.

\section{Data recorded}

Potato tubers were harvested when they attained edible stage. Six square meter area from each plot was harvested to record tuber and haulm yields. The weights of tuber were taken just after harvest. The haulm yield was expressed on sundry basis. Data on plant height were recorded from 10 randomly selected representative plants from outside the harvested area within a plot, as described by Gomez and Gomez (1984).

\section{Collection and preparation of plant samples for chemical analysis}

Plant samples (tuber and haulm) were collected at the time of harvesting. The haulm samples were air dried immediately after collection and the dry samples were chopped off into smaller pieces. The collected plant samples were then oven dried at $65^{\circ} \mathrm{C}$ for 24 hours. To obtain homogenous powder, the samples were finely ground by using a grinding-mill to pass through a 60-mesh sieve. The processed plant samples were chemically analyzed for determination of $\mathrm{N}, \mathrm{P}, \mathrm{K}, \mathrm{S}, \mathrm{Zn}$ and $\mathrm{B}$ concentrations following the methods stated in Table 3. Nutrient uptake was calculated from the yield and respective nutrient concentration data using the following formulae-

-For N, P, K and S: 
Nutrient uptake by tuber $\left(\mathrm{kg} \mathrm{ha}^{-1}\right)=$ Nutrient content in tuber $(\%) \times$ Oven dry yield $(\mathrm{t}$ $\left.\mathrm{ha}^{-1}\right) \times 10$

Nutrient uptake by haulm $\left(\mathrm{kg} \mathrm{ha}^{-1}\right)=$ Nutrient content in haulm $(\%) \times$ Oven dry yield $\left(\mathrm{kg} \mathrm{ha}^{-1}\right) / 100$

-For $\mathrm{Zn}$ and $\mathrm{B}$ :

Nutrient uptake $\left(\mathrm{g} \mathrm{ha}^{-1}\right)=$ Nutrient content in tuber $\left(\mu \mathrm{gg}^{-1}\right) \times$ Oven dry yield $\left(\mathrm{t} \mathrm{ha}^{-1}\right)$

Nutrient uptake $\left(\mathrm{g} \mathrm{ha}^{-1}\right)=$ Nutrient content in haulm $\left(\mu \mathrm{g} \mathrm{g}^{-1}\right) \times$ Yield $\left(\mathrm{kg} \mathrm{ha}^{-1}\right) / 1000$

Protein concentrations of potato tuber were calculated from $\mathrm{N}$ concentration of tuber by using the following formulae-

$\%$ Protein $=\% \mathrm{~N}$ of the produce $\times 6.25 .(\mathrm{FAO} / \mathrm{WHO} / \mathrm{UNU}, 1985)$

Table 2. Methods used for plant analysis

\begin{tabular}{cll}
\hline $\begin{array}{c}\text { Elements } \\
\text { analysed }\end{array}$ & Analytical methods used \\
\hline $\mathrm{N}$ & Micro-Kjeldahl method (Bremner and Mulvaney, 1982) \\
$\mathrm{P}$ & Colorimetric method (Yoshida et al., 1976). \\
$\mathrm{K}$ & $\begin{array}{l}\text { Ammonium acetate extraction method using flame photometer (Yoshida } \text { et } \\
\text { al., 1976) }\end{array}$ \\
$\mathrm{S}$ & Turbidimetric method (Chapman and Pratt, 1961) \\
$\mathrm{Zn}$ & $\begin{array}{l}\text { DTPA extraction method using atomic adsorption spectrophotometer } \\
\text { (Yoshida } \text { et al., 1976) } \\
\mathrm{B}\end{array}$ & $\begin{array}{l}\text { The B concentration was determined by spectrophotometer following } \\
\text { azomethine-H method (Keren, 1996) }\end{array}$ \\
&
\end{tabular}

\section{Data analysis}

The collected data were compiled and tabulated, which were subjected to statistical analyses following standard methodology and the mean differences were adjudged by Duncan's Multiple Range Test (Gomez and Gomez, 1984).

\section{RESULTS}

The effects were evaluated in terms of tuber yield, haulm yield, plant height, protein $\&$ zinc concentration of tuber, and nutrient uptake by tuber $\&$ haulm.

\section{Plant height}

Plant height of potato was significantly affected by the treatments showing a range of 45.2-56.7 cm (Table 3). The highest plant height was observed in $\mathrm{T}_{7}$ treatment which had statistical similarities to those of all other treatments except control. 


\section{Tuber yield}

Tuber yield of potato was significantly influenced by the micronutrients treatment (Table 3). The highest tuber yield $\left(30.3 \mathrm{t} \mathrm{ha}^{-1}\right)$ was recorded in $\mathrm{T}_{6}$ treatment, which was statistically similar to those of all other treatments except $T_{1}$ and $T_{2}$. The lowest tuber yield $\left(21.0 \mathrm{t} \mathrm{ha}^{-1}\right)$ was produced by control treatment. The $\mathrm{T}_{2}$ treatment produced tuber yield $\left(25.3 \mathrm{tha}^{-1}\right)$, which was higher than that of control but similar to $\mathrm{T}_{3}$ and $\mathrm{T}_{4}$ treatments. Like tuber yield, haulm yield was also significantly influenced by the treatments and it varied from $9.64 \mathrm{~kg} \mathrm{ha}^{-1}$ in $\mathrm{T}_{1}$ to $1.23 \mathrm{~kg} \mathrm{ha}^{-1}$ in $\mathrm{T}_{7}$ treatment. All treatments other than control produced statistically similar haulm yield.

\section{Protein content of potato tuber}

Nutritional quality of potato tuber with regards to protein and zinc concentrations varied significantly due to application of different micronutrients (Table 4). Protein concentration ranged from $1.31 \%$ in control treatment to $1.81 \%$ in $\mathrm{T}_{5}$ treatment. Except control, all treatment effects had statistical similarities to each other which indicated that addition of micronutrients other than $\mathrm{Zn}$ did not add any extra benefit. It is also noted that $\mathrm{Zn}$ helped protein synthesis in tuber, which improves food quality.

Table 3. Effects of micronutrients on plant height and yield of potato

\begin{tabular}{l|c|c|c}
\hline \multicolumn{1}{c|}{ Treatments } & $\begin{array}{c}\text { Plant height } \\
(\mathrm{cm})\end{array}$ & $\begin{array}{c}\text { Tuber yield } \\
\left(\mathrm{t} \mathrm{ha}^{-1}\right)\end{array}$ & $\begin{array}{c}\text { Haulm yield } \\
\left(\mathrm{t} \mathrm{ha}^{-1}\right)\end{array}$ \\
\hline $\mathrm{T}_{1}:$ Control & $45.2 \mathrm{~b}$ & $21.0 \mathrm{c}$ & $9.64 \mathrm{~b}$ \\
$\mathrm{~T}_{2}: \mathrm{Zn}$ & $56.7 \mathrm{a}$ & $25.3 \mathrm{~b}$ & $1.18 \mathrm{a}$ \\
$\mathrm{T}_{3}: \mathrm{Zn}+\mathrm{B}$ & $56.3 \mathrm{a}$ & $28.7 \mathrm{ab}$ & $1.18 \mathrm{a}$ \\
$\mathrm{T}_{4}: \mathrm{Zn}+\mathrm{B}+\mathrm{Cu}$ & $55.0 \mathrm{a}$ & $28.9 \mathrm{ab}$ & $1.16 \mathrm{a}$ \\
$\mathrm{T}_{5}: \mathrm{Zn}+\mathrm{B}+\mathrm{Cu}+\mathrm{Mn}$ & $55.8 \mathrm{a}$ & $29.5 \mathrm{a}$ & $1.18 \mathrm{a}$ \\
$\mathrm{T}_{6}: \mathrm{Zn}+\mathrm{B}+\mathrm{Cu}+\mathrm{Mn}+\mathrm{Fe}$ & $55.8 \mathrm{a}$ & $30.3 \mathrm{a}$ & $1.20 \mathrm{a}$ \\
$\mathrm{T}_{7}: \mathrm{Zn}+\mathrm{B}+\mathrm{Cu}+\mathrm{Mn}+\mathrm{Fe}+\mathrm{Mo}$ & $56.7 \mathrm{a}$ & $29.8 \mathrm{a}$ & $1.23 \mathrm{a}$ \\
\hline $\mathrm{CV}(\%)$ & 5.23 & 7.79 & 5.92 \\
\hline Significance level & $* *$ & $* *$ & $* *$ \\
$\mathrm{SE}( \pm)$ & 1.65 & 1.24 & 0.04 \\
\hline
\end{tabular}

Means followed by same letter in a column are not significantly different at $5 \%$ level by DMRT.

$\mathrm{SE}( \pm)=$ Standard error of means, $\mathrm{CV}=$ Co-efficient of variation, $* *=$ Significant at $1 \%$ level 
Table 4. Effects of micronutrients on protein and zinc concentrations of potato tuber

\begin{tabular}{lc}
\hline Treatments & Protein $(\%)$ \\
\hline $\mathrm{T}_{1}:$ Control & $1.31 \mathrm{~b}$ \\
$\mathrm{~T}_{2}: \mathrm{Zn}$ & $1.64 \mathrm{a}$ \\
$\mathrm{T}_{3}: \mathrm{Zn}+\mathrm{B}$ & $1.68 \mathrm{a}$ \\
$\mathrm{T}_{4}: \mathrm{Zn}+\mathrm{B}+\mathrm{Cu}$ & $1.67 \mathrm{a}$ \\
$\mathrm{T}_{5}: \mathrm{Zn}+\mathrm{B}+\mathrm{Cu}+\mathrm{Mn}$ & $1.81 \mathrm{a}$ \\
$\mathrm{T}_{6}: \mathrm{Zn}+\mathrm{B}+\mathrm{Cu}+\mathrm{Mn}+\mathrm{Fe}$ & $1.66 \mathrm{a}$ \\
$\mathrm{T}_{7}: \mathrm{Zn}+\mathrm{B}+\mathrm{Cu}+\mathrm{Mn}+\mathrm{Fe}+\mathrm{Mo}$ & $1.67 \mathrm{a}$ \\
\hline $\mathrm{CV}(\%)$ & 9.15 \\
\hline Significance level & $*$ \\
$\mathrm{SE}( \pm)$ & 0.09 \\
\hline
\end{tabular}

Note: Protein and zinc concentration of potato tuber is expressed as fresh weight basis.

Means followed by same letter in a column are not significantly different at $5 \%$ level by DMRT.

SE $( \pm)=$ Standard error of means, CV $=$ Co-efficient of variation, $*=$ Significant at $5 \%$ level

\section{Nutrient concentration and their uptake by potato}

Nutrient concentrations of potato tuber were expressed as fresh weight basis (Table 5). Other than $P$ in tuber and $S$ in both tuber and haulm, the nutrient concentrations of potato affected significantly by the treatments applied. In almost all cases (except $\mathrm{K}$ in tuber and B in both tuber and haulm), application of only $\mathrm{Zn}$ was found to be sufficient for the highest concentrations. The uptake of different nutrient elements as calculated from respective nutrient concentration and yield data is presented in Table 6 and discussed below :-

\section{Nitrogen uptake}

There were significant differences in the $\mathrm{N}$ uptake by potato (tuber + haulm) due to different treatments. The highest uptake of tuber $\mathrm{N}$ and total uptake of $\mathrm{N}$ (86.3 and $107 \mathrm{~kg} \mathrm{ha}^{-1}$, respectively) was found in $\mathrm{T}_{5}(\mathrm{Zn}+\mathrm{B}+\mathrm{Cu}+\mathrm{Mn})$ treatment, while that of haulm $\mathrm{N}\left(22.1 \mathrm{~kg} \mathrm{ha}^{-1}\right)$ was in $\mathrm{T}_{7}(\mathrm{Zn}+\mathrm{B}+\mathrm{Cu}+\mathrm{Mn}+\mathrm{Fe}+\mathrm{Mo})$ treatment. Except $\mathrm{T}_{1}$, all other treatments had statistically similar effects on $\mathrm{N}$ uptake. 
Table 5. Effects of micronutrients on the nutrient concentration of potato

\begin{tabular}{|c|c|c|c|c|c|c|c|c|c|c|c|c|}
\hline \multirow[t]{2}{*}{ Treatments } & \multicolumn{2}{|c|}{$\begin{array}{c}\mathrm{N} \\
(\%)\end{array}$} & \multicolumn{2}{|c|}{$\begin{array}{c}\mathrm{P} \\
(\%)\end{array}$} & \multicolumn{2}{|c|}{$\begin{array}{c}\mathrm{K} \\
(\%)\end{array}$} & \multicolumn{2}{|c|}{$\begin{array}{c}\mathrm{S} \\
(\%)\end{array}$} & \multicolumn{2}{|c|}{$\begin{array}{c}\mathrm{Zn} \\
\left(\mu \mathrm{g} \mathrm{g}^{-1}\right)\end{array}$} & \multicolumn{2}{|c|}{$\begin{array}{c}\mathrm{B} \\
\left(\mu \mathrm{g} \mathrm{g}^{-1}\right) \\
\end{array}$} \\
\hline & Tuber & Haulm & Tuber & Haulm & Tuber & Haulm & Tuber & Haulm & Tuber & Haulm & Tuber & Haulm \\
\hline $\mathrm{T}_{1}$ : Control & $0.209 \mathrm{~b}$ & $1.56 \mathrm{~b}$ & 0.063 & $0.214 \mathrm{a}$ & $0.414 \mathrm{c}$ & $1.66 \mathrm{~b}$ & 0.044 & 0.207 & $6.98 \mathrm{~b}$ & $85 \mathrm{~b}$ & $6.71 \mathrm{~b}$ & $37.2 b$ \\
\hline $\mathrm{T}_{2}: \mathrm{Zn}$ & $0.263 \mathrm{a}$ & $1.77 \mathrm{a}$ & 0.052 & $0.180 \mathrm{c}$ & $0.469 \mathrm{~b}$ & $1.90 \mathrm{a}$ & 0.051 & 0.238 & $8.56 \mathrm{a}$ & $102 \mathrm{a}$ & $7.29 \mathrm{~b}$ & $39.5 b$ \\
\hline $\mathrm{T}_{3}: \mathrm{Zn}+\mathrm{B}$ & $0.269 \mathrm{a}$ & $1.79 \mathrm{a}$ & 0.052 & $0.204 \mathrm{ab}$ & $0.520 \mathrm{a}$ & $1.87 \mathrm{a}$ & 0.050 & 0.235 & $8.60 \mathrm{a}$ & $108 \mathrm{a}$ & $8.86 \mathrm{a}$ & $53.0 \mathrm{a}$ \\
\hline $\mathrm{T}_{4}: \mathrm{Zn}+\mathrm{B}+\mathrm{Cu}$ & $0.267 \mathrm{a}$ & $1.81 \mathrm{a}$ & 0.053 & $0.193 b c$ & $0.515 \mathrm{a}$ & $1.85 \mathrm{a}$ & 0.049 & 0.220 & $8.45 \mathrm{a}$ & $107 \mathrm{a}$ & $8.54 \mathrm{a}$ & $51.6 \mathrm{a}$ \\
\hline $\mathrm{T}_{5}: \mathrm{Zn}+\mathrm{B}+\mathrm{Cu}+\mathrm{Mn}$ & $0.289 \mathrm{a}$ & $1.77 \mathrm{a}$ & 0.055 & $\mathrm{ab}$ & 0 . & $1.90 \mathrm{a}$ & 0.050 & 0.232 & $8.27 \mathrm{a}$ & $107 \mathrm{a}$ & $8.73 \mathrm{a}$ & $49.9 \mathrm{a}$ \\
\hline $\mathrm{T}_{6}: \mathrm{Zn}+\mathrm{B}+\mathrm{Cu}+\mathrm{Mn}+\mathrm{Fe}$ & $0.266 \mathrm{a}$ & $1.78 \mathrm{a}$ & 0.054 & $0.190 b c$ & $0.511 \mathrm{a}$ & $1.91 \mathrm{a}$ & 0.050 & 0.232 & $8.64 \mathrm{a}$ & $109 a$ & $8.88 \mathrm{a}$ & $53.7 \mathrm{a}$ \\
\hline $\begin{array}{l}\mathrm{T}_{7}: \\
\mathrm{Zn}+\mathrm{B}+\mathrm{Cu}+\mathrm{Mn}+\mathrm{Fe}+\mathrm{Mo}\end{array}$ & $0.267 \mathrm{a}$ & $1.80 \mathrm{a}$ & 0.053 & $0.212 \mathrm{a}$ & $0.505 \mathrm{a}$ & $1.89 \mathrm{a}$ & 0.050 & 0.228 & $8.51 \mathrm{a}$ & $108 \mathrm{a}$ & $8.59 \mathrm{a}$ & $52.4 \mathrm{a}$ \\
\hline $\mathrm{CV}(\%)$ & 9.12 & 2.34 & 8.69 & 4.90 & 3.80 & 2.43 & 5.91 & 7.86 & 6.21 & 7.86 & 6.81 & 7.74 \\
\hline Significance level & $*$ & $* *$ & NS & $* *$ & $* *$ & $* *$ & NS & NS & $*$ & $*$ & $* *$ & $* *$ \\
\hline $\mathrm{SE}( \pm)$ & 0.014 & 0.024 & 0.003 & 0.006 & 0.011 & 0.026 & 0.002 & 0.011 & 0.30 & 4.71 & 0.32 & 2.15 \\
\hline
\end{tabular}

Note: Nutrient concentration of potato tuber was expressed as fresh weight basis.

Means followed by same letter in a column are not significantly different at $5 \%$ level by DMRT

$\mathrm{SE}( \pm)=$ Standard error of means, $\mathrm{CV}=\mathrm{Co}$-efficient of variation,

$*=$ Significant at $5 \%$ level, $* *=$ Significant at $1 \%$ level, NS=Non-significant

Table 6 Effects of micronutrients on the nutrient uptake of potato

\begin{tabular}{l|c|c|c|c|c|c|c|c|c}
\hline \multirow{2}{*}{ Treatments } & \multicolumn{3}{c|}{$\mathrm{N}$ uptake $\left(\mathrm{kg} \mathrm{ha}^{-1}\right)$} & \multicolumn{3}{c|}{ P uptake $\left(\mathrm{kg} \mathrm{ha}^{-1}\right)$} & \multicolumn{3}{c}{ K uptake $\left(\mathrm{kg} \mathrm{ha}^{-1}\right)$} \\
\cline { 2 - 10 } & Tuber & Haulm & Total & Tuber & Haulm & Total & Tuber & Haulm & Total \\
\hline $\mathrm{T}_{1}:$ Control & $43.7 \mathrm{~b}$ & $15.1 \mathrm{~b}$ & $59 \mathrm{~b}$ & 13.3 & $2.06 \mathrm{~d}$ & 15.3 & $87 \mathrm{c}$ & $16.0 \mathrm{~b}$ & $103 \mathrm{c}$ \\
$\mathrm{T}_{2}: \mathrm{Zn}$ & $66.5 \mathrm{a}$ & $20.9 \mathrm{a}$ & $87 \mathrm{a}$ & 13.1 & $2.12 \mathrm{~cd}$ & 15.2 & $119 \mathrm{~b}$ & $22.3 \mathrm{a}$ & $141 \mathrm{~b}$ \\
$\mathrm{~T}_{3}: \mathrm{Zn}+\mathrm{B}$ & $77.0 \mathrm{a}$ & $21.2 \mathrm{a}$ & $98 \mathrm{a}$ & 14.9 & $2.42 \mathrm{abc}$ & 17.3 & $149 \mathrm{a}$ & $22.1 \mathrm{a}$ & $171 \mathrm{a}$ \\
$\mathrm{T}_{4}: \mathrm{Zn}+\mathrm{B}+\mathrm{Cu}$ & $77.2 \mathrm{a}$ & $21.0 \mathrm{a}$ & $98 \mathrm{a}$ & 15.4 & $2.24 \mathrm{bcd}$ & 17.7 & $149 \mathrm{a}$ & $21.5 \mathrm{a}$ & $170 \mathrm{a}$ \\
$\mathrm{T}_{5}: \mathrm{Zn}+\mathrm{B}+\mathrm{Cu}+\mathrm{Mn}$ & $86.3 \mathrm{a}$ & $20.9 \mathrm{a}$ & $107 \mathrm{a}$ & 16.3 & $2.46 \mathrm{ab}$ & 18.7 & $148 \mathrm{a}$ & $22.4 \mathrm{a}$ & $171 \mathrm{a}$ \\
$\mathrm{T}_{6}: \mathrm{Zn}+\mathrm{B}+\mathrm{Cu}+\mathrm{Mn}+\mathrm{Fe}$ & $80.6 \mathrm{a}$ & $21.4 \mathrm{a}$ & $102 \mathrm{a}$ & 16.5 & $2.29 \mathrm{bcd}$ & 18.8 & $155 \mathrm{a}$ & $23.0 \mathrm{a}$ & $178 \mathrm{a}$ \\
$\mathrm{T}_{7}: \mathrm{Zn}+\mathrm{B}+\mathrm{Cu}+\mathrm{Mn}+\mathrm{Fe}+\mathrm{Mc}$ & $79.6 \mathrm{a}$ & $22.1 \mathrm{a}$ & $102 \mathrm{a}$ & 16.0 & $2.61 \mathrm{a}$ & 18.6 & $151 \mathrm{a}$ & $23.2 \mathrm{a}$ & $174 \mathrm{a}$ \\
$\mathrm{CV}(\%)$ & 14.7 & 6.23 & 11.4 & 14.8 & 7.05 & 13.3 & 10.7 & 6.5 & 9.6 \\
$\mathrm{Significance} \mathrm{level}$ & $* *$ & $* *$ & $* *$ & NS & $* *$ & NS & $* *$ & $* *$ & $* *$ \\
$\mathrm{SE}( \pm)$ & 6.21 & 0.73 & 6.15 & 1.29 & 0.02 & 1.33 & 8.5 & 0.81 & 8.8 \\
$\mathrm{~T}_{1}: \mathrm{Control}$ & $9.3 \mathrm{~b}$ & $1.98 \mathrm{~b}$ & $11.3 \mathrm{~b}$ & $146 \mathrm{c}$ & $83 \mathrm{~b}$ & $229 \mathrm{c}$ & $141 \mathrm{~b}$ & $35.6 \mathrm{c}$ & $177 \mathrm{~b}$ \\
$\mathrm{~T}_{2}: \mathrm{Zn}$ & $12.9 \mathrm{a}$ & $2.81 \mathrm{a}$ & $15.7 \mathrm{a}$ & $217 \mathrm{~b}$ & $120 \mathrm{a}$ & $337 \mathrm{~b}$ & $184 \mathrm{~b}$ & $46.6 \mathrm{~b}$ & $231 \mathrm{~b}$ \\
$\mathrm{~T}_{3}: \mathrm{Zn}+\mathrm{B}$ & $14.3 \mathrm{a}$ & $2.78 \mathrm{a}$ & $17.1 \mathrm{a}$ & $246 \mathrm{ab}$ & $128 \mathrm{a}$ & $374 \mathrm{ab}$ & $254 \mathrm{a}$ & $62.8 \mathrm{a}$ & $316 \mathrm{a}$ \\
$\mathrm{T}_{4}: \mathrm{Zn}+\mathrm{B}+\mathrm{Cu}$ & $14.1 \mathrm{a}$ & $2.55 \mathrm{a}$ & $16.6 \mathrm{a}$ & $243 \mathrm{ab}$ & $125 \mathrm{a}$ & $369 \mathrm{ab}$ & $247 \mathrm{a}$ & $59.9 \mathrm{a}$ & $306 \mathrm{a}$ \\
\hline
\end{tabular}




\begin{tabular}{l|c|c|c|c|c|c|cc|c}
\hline \multirow{2}{*}{ Treatments } & \multicolumn{3}{c|}{ N uptake $\left(\mathrm{kg} \mathrm{ha}^{-1}\right)$} & \multicolumn{3}{c|}{ P uptake $\left(\mathrm{kg} \mathrm{ha}^{-1}\right)$} & \multicolumn{3}{c}{ K uptake $\left(\mathrm{kg} \mathrm{ha}^{-1}\right)$} \\
\cline { 2 - 10 } & Tuber & Haulm & Total & Tuber & Haulm & Total & Tuber & Haulm & Total \\
\hline $\mathrm{T}_{5}: \mathrm{Zn}+\mathrm{B}+\mathrm{Cu}+\mathrm{Mn}$ & $14.7 \mathrm{a}$ & $2.74 \mathrm{a}$ & $17.4 \mathrm{a}$ & $241 \mathrm{ab}$ & $126 \mathrm{a}$ & $367 \mathrm{ab}$ & $258 \mathrm{a}$ & $58.7 \mathrm{a}$ & $317 \mathrm{a}$ \\
$\mathrm{T}_{6}: \mathrm{Zn}+\mathrm{B}+\mathrm{Cu}+\mathrm{Mn}+\mathrm{Fe}$ & $15.2 \mathrm{a}$ & $2.80 \mathrm{a}$ & $18.0 \mathrm{a}$ & $263 \mathrm{a}$ & $132 \mathrm{a}$ & $395 \mathrm{a}$ & $270 \mathrm{a}$ & $64.9 \mathrm{a}$ & $335 \mathrm{a}$ \\
$\mathrm{T}_{7}: \mathrm{Zn}+\mathrm{B}+\mathrm{Cu}+\mathrm{Mn}+\mathrm{Fe}+\mathrm{Mc}$ & $14.9 \mathrm{a}$ & $2.80 \mathrm{a}$ & $17.7 \mathrm{a}$ & $254 \mathrm{ab}$ & $133 \mathrm{a}$ & $387 \mathrm{ab}$ & $257 \mathrm{a}$ & $64.6 \mathrm{a}$ & $321 \mathrm{a}$ \\
$\mathrm{CV}(\%)$ & 11.6 & 9.70 & 10.8 & 9.30 & 11.3 & 8.3 & 12.5 & 10.5 & 11.5 \\
Significance level & $* *$ & $*$ & $* *$ & $* *$ & $* *$ & $* *$ & $* *$ & $* *$ & $* *$ \\
$\mathrm{SE}( \pm)$ & 0.91 & 0.02 & 1.02 & 12.4 & 7.9 & 16.8 & 16.6 & 3.42 & 19.0 \\
\hline
\end{tabular}

Means followed by same letter in a column are not significantly different at $5 \%$ level by DMRT.

SE $( \pm)=$ Standard error of means, CV $=$ Co-efficient of variation, $*=$ Significant at $5 \%$ level, $* *=$ Significant at $1 \%$ level, NS=Non-significant

\section{Phosphorus uptake}

Total uptake of $\mathrm{P}$ as well as tuber $\mathrm{P}$ uptake did not vary significantly with the micronutrient treatments but haulm uptake of $\mathrm{P}$ was affected. Tuber $\mathrm{P}$ uptake ranged from $13.1 \mathrm{~kg} \mathrm{ha}^{-1}$ in $\mathrm{T}_{2}$ to $16.5 \mathrm{~kg} \mathrm{ha}^{-1}$ in $\mathrm{T}_{6}$ treatment, while the haulm $\mathrm{P}$ uptake varied from $2.06 \mathrm{~kg} \mathrm{ha}^{-1}$ in $\mathrm{T}_{1}$ to $2.61 \mathrm{~kg} \mathrm{ha}^{-1}$ in $\mathrm{T}_{7}$ treatment. The highest $\mathrm{P}$ uptake of $18.8 \mathrm{~kg} \mathrm{ha}^{-1}$ (tuber + haulm) was recorded for $\mathrm{T}_{6}$ treatment and the lowest $\mathrm{P}$ uptake $\left(15.2 \mathrm{~kg} \mathrm{ha}^{-1}\right)$ was due to $\mathrm{T}_{2}$ treatment. It was noted that, tuber $\mathrm{P}$ uptake in $\mathrm{T}_{1}$ was higher than that in $\mathrm{T}_{2}$ treatment. Such exception was due to the higher $\mathrm{P}$ concentration in $\mathrm{T}_{1}$ as compared to $\mathrm{T}_{2}$ treatment.

\section{Potassium uptake}

Uptake of $\mathrm{K}$ by potato significantly increased due to application of micronutrients to soil. The highest uptake of tuber $\mathrm{K}$ and total uptake of $\mathrm{K}\left(155\right.$ and $178 \mathrm{~kg} \mathrm{ha}^{-1}$, respectively) was observed in $\mathrm{T}_{6}$ treatment and the highest haulm $\mathrm{K}$ uptake $(23.2 \mathrm{~kg}$ $\mathrm{ha}^{-1}$ ) was recorded in $\mathrm{T}_{7}$ treatment. The treatment having the highest $\mathrm{K}$ uptake by tuber and the highest total $\mathrm{K}$ uptake was statistically identical with all other treatments except $\mathrm{T}_{1}$ and $\mathrm{T}_{2}$.

\section{Sulphur uptake}

The $\mathrm{S}$ uptake by potato was also affected significantly by the various micronutrient treatments. The highest uptake of tuber $S$ and haulm $S$ (15.21 and $2.81 \mathrm{~kg} \mathrm{ha}^{-1}$, respectively) was found in $\mathrm{T}_{6}$ and $\mathrm{T}_{2}$ treatments.

\section{Zinc uptake}

The $\mathrm{Zn}$ uptake by potato was significantly influenced by the treatments used. The highest uptake of tuber and total Zn (263 and $395 \mathrm{~g} \mathrm{ha}^{-1}$, respectively) was observed in $T_{6}$ treatment and it was statistically similar to all other treatments except $T_{1}$ and $T_{2}$. On the other hand, $\mathrm{Zn}$ uptake in haulm was the highest $\left(133 \mathrm{~g} \mathrm{ha}^{-1}\right)$ in $\mathrm{T}_{7}$ treatment which was statistically similar to those of all other treatments except $T_{1}$. 


\section{Boron uptake}

Like zinc uptake, boron uptake by potato also significantly increased over the control as an effect of different micronutrient treatments. The highest uptake of 270 and 64.9 $\mathrm{g} \mathrm{ha}^{-1}$ by tuber and haulm, respectively, was observed in $\mathrm{T}_{6}$ treatment which was statistically similar to that recorded with all other treatments except $\mathrm{T}_{1}$ and $\mathrm{T}_{2}$.

\section{DISCUSSION}

The effect of micronutrients application on different traits of potato was studied through field trials followed by chemical analysis in the laboratory. Plant height at harvest was affected significantly due to application of different treatments. Only $\mathrm{Zn}$ was found to affect plant height. Pregno and Armour (1992) stated that application of B did not increase plant height of potato. Tuber yield of potato was significantly influenced by different micronutrient treatments. Tuber yield increased significantly by the application of $\mathrm{Zn}$ but to obtain the highest yield B was needed to apply. This result has similarities with the findings of some other scientists. Dwivedi (1991) showed that $\mathrm{ZnSO}_{4}$ applications can increase potato yield by $37 \%$ and spraying with $\mathrm{Zn}$ increased potato yield. Trehan and Grewal (1981) stated that in potato cultivation, $\mathrm{Zn}$ and $\mathrm{B}$ can help in increasing the foliage coverage at initial growth stages and in the later stages, the translocation of assimilates is responsible for higher yield. Chaudhary et al. (2001) found that potato responded quadratically to $\mathrm{Zn}$ application. Puzina (2004) observed that potato fertilization using boric acid caused an increase in tuber size and weight by increasing of cell diameter in the tuber.

Like tuber yield, application of only $\mathrm{Zn}$ was sufficient to achieve the statistically highest haulm yield. The application of $20 \mathrm{ppm} \mathrm{Zn}$ increased the dry weight of stem, root and main stolen (Langille and Batteese 1974). Bari et al. (2001) showed that application of $1.1 \mathrm{~kg} \mathrm{~B} \mathrm{ha}^{-1}$ from borax increased potato fresh haulm weight hill ${ }^{-1}$ and yield of tuber ha ${ }^{-1}$. Considering nutritional aspects, protein contents of vegetables is a matter of concern in the present situation. In this study, only Zn was sufficient to achieve the highest protein concentration of potato tuber. According to Mousavi et al. (2012) zinc is essential micronutrients for protein production in plants; also zinc is main composition of ribosome and is essential for their development. Zinc is active element in biochemical processes and has a chemical and biological interaction with some other elements. Phosphorus is the most important element which interferes on zinc uptake by plants. Micronutrient application influenced the uptake of N, K, S, Zn and $\mathrm{B}$ by potato. $\mathrm{P}$ uptake by the crops did not affect significantly. This might be due to antagonistic relationship between $\mathrm{Zn}$ and $\mathrm{P}$ in soil-plant system. Except $\mathrm{Zn}$ and B, the other micronutrients did not play positive role on nutrient uptake by the crops. Sandeep et al. (2014) reported that application B and Zn enhanced potato tuber yield and also influenced uptake of N, P, K, S, Zn and B. El-Banna and Abd El-Salam (2005) reported that foliar spraying of potato plants with B at $75 \mathrm{ppm}$ significantly recorded the highest concentrations of N, K and B in plants. Arisha et al. (1999) 
found that foliar spray of B as boric acid with recommended doses of NPK increased concentration and total uptake of N, P and K. El-Mahdy (2007) showed that foliar spray of $\mathrm{B}$ at rate $75 \mathrm{ppm}$ increased concentration of $\mathrm{N}, \mathrm{P}, \mathrm{K}$ and $\mathrm{B}$ and its uptake.

\section{CONCLUSION}

In floodplain soil application of zinc and boron fertilizers can help to boost up potato yield as well as ensure nutritional concerns. It is needed to conduct further exclusive research to optimize application rates of these nutrients to potato in floodplain soil.

\section{ACKNOWLEDGEMENT}

The authors gratefully acknowledge the financial support provided by Coordinated Project on Soil Fertility and Fertilizer Management for Crops and Cropping Patterns (BAU component), NATP, BARC during the research period.

\section{REFERENCES}

Alam, M.S, Islam, N., and Jahiruddin, M. (2000). Effects of zinc and boron application on the performances of local and hybrid maize, Bangladesh Journal of Soil Science, 26, 95101.

Arisha, H.M., El-Ghamriny, E.A., and Nour, K.A. (1999). Studies on tomato flowering, fruit set, yield and quality in summer season. Spraying with boron, zinc and phosphorus. Zagazig Journal of Agricultural Research, 26(5), 1365-1384.

Bari, M.S., Rabbani, M.G., Rahman, M.Sq., Islam, M.J., and Hoque, A.T.M.R. (2001). Effect of zinc, boron, sulphur and magnesium on the growth and yield of potato. Pakistan Journal of Biological Science, 4(9), 1090-1093.

BBS. (2017). Bangladesh Bureau of Statistics. The Year Book of Agricultural Statistics of Bangladesh. Statistics and Informatics Division, Ministry of Planning, Government of the People's Republic of Bangladesh, Dhaka. P. 360.

Bell, R.W. and Dell, B. (2008). Micronutrients for sustainable food, feed, fibre and bioenergy production. International Fertilizer Industry Association (IFA), Paris, France.

Bhuiyan, M.A.H., Khanam, D., Khatun, M.R., and Hassan, M.S. (1998). Effects of molybdenum, boron and Rhizobium on nodulation, growth and yield of chickpea, Bulletin Institute of Tropical Agriculture Kyushu University, 21, 1-7.

Chaudhary, R.A., Akram, M, Gill, K.H., and Qazi, M.A. (2001). Zinc requirement of potato crop in the Punjab. Pakistan Journal of Soil Science, 19(1-2), 81-83.

Dwivedi, G.K. (1991). Mode of application of micronutrients to potato in acid soil for Garhwal Himalaya. Indian Journal of Horticulture, 45, 258-263.

El-Banna, E.N. and El-Salam, H.Z.A. (2005). Response of potato plants for different sources of potassium with different foliar rates of boron and molybdenum. Journal Agricultural Science, 30(10), 6221-6233.

El-Mahdy, R.E. (2007). Effect of heavy nitrogen application on pepper plant (Capsicum annuит). M. Sci. Thesis, Faculty of Agriculture. Mansoura University, Egypt. 
FAO/WHO/UNU. (1985). Energy and protein requirements, Report of a joint FAO/WHO/UNU expert consultation, WHO Technical Report Series 724, Geneva, WHO.

FRG. (2005). Fertilizer Recommendation Guide. Bangladesh Agricultural Research Council (BARC), Dhaka, Bangladesh.

Gomez, K.A., and Gomez, A.A. (1984). Statistical Procedures for Agricultural Research, John Wiley and Sons, New York.

Hossain, M.S. (2005). Effects of Different Methods of Zinc Application on Grain Yield and Grain Zinc Concentration of Wheat Genotypes, MS Thesis, Department of Soil Science, Bangladesh Agricultural University, Mymensingh, Bangladesh.

Islam, M.S. (2008). Soil fertility history, present status and future scenario in Bangladesh, Bangladesh Journal of Agriculture and Environment, 4, 129-151.

Jahiruddin, M., and Islam, M.R. (2014). Project Report (2011-2014). Requirement of Micronutrients for Crops and Cropping Patterns. PIU-BARC (NATP Phase-I) project. Project ID No. 339.

Jahiruddin, M., Islam, M.N., Hashem, M.A. and Islam, M.R. (1994). Influence of sulphur, zinc and boron on yield and nutrient uptake of BR2 rice. Progressive Agriculture, 5(1), 61-67.

Khanam, R., Arefin, M.S., Haque, M.A., Islam, M.R., and Jahiruddin, M. (2000). Effects of magnesium, boron, and molybdenum on the growth, yield and protein content of chickpea and lentil, Progressive Agriculture, 11(1\&2), 77-80.

Langille, B., and Batteese, I.R. (1974). Influence of manganese concentration in nutrient solution on growth and elemental content of the katahdin potato plant. Canadian Journal of Plant Science, 54, 375-381.

Mousavi, S.R., Galavi, M., and Rezaei, M. (2012). The interaction of zinc with other elements in plants: a review, International Journal of Agriculture and Crop Science, 4(24), 1881-1884.

Nasreen, S., Siddiky, M.A., Ahmed, R., and Rahman, M.H. (2009). Response of okra to boron and zinc fertilization. Presented at the research review and programme planning workshop on soils programme of NARS institutes, BARC, Dhaka.

Pregno, L.M., and Armour, J.D. (1992). Boron deficiency and toxicity in potato cv. Sebago on an oxisol of the Atherton, North Queensland. Australian Journal of Experimental Agriculture, 32, 251-253.

Puzina, T.I. (2004). Effect of zinc sulphate and boric acid on the hormonal status of potato plants in relation to tuberization. Russian Journal Plant Phisiology, 51(2), 209-215.

Rijpma, J., and Jahiruddin, M. (2004). Strategy and plan for use of soil nutrient balance in Bangladesh, Final Report of Short-term Assignment. SFFP/DANIDA.

Sandeep, S., Deependra, K., Singh, C.B., and Vinay, S. (2014). Effect of balanced fertilization on yield, nutrients uptake and economics of potato (Solanum tuberosum) in alluvial soil. Indian Journal of Agronomy, 59(30), 451-454.

Trehan, S.P. and Grewal, J.S. (1981). Comparative efficiency of methods of application of zinc to potato. Indian Journal of Agricultural Science, 51, 240-243.

Upadhayay, N.C., Singh, B.P., Kaushik, S.K., Khan, M.A., Ezekiel, R., Singh, N., and Kumar M. (2012). Zinc deficiency in potato tubers, CPRI Newsletter, Central Potato Research Institute, Shimla 171 001, HP, India, pp. 1-2. 